\title{
Structure of the Book
}

This book is composed of seven modules. The first four modules examine various aspects of translating different genres into Arabic. A range of drills then follows, which are designed to help learners digest these concepts. Modules 5, 6 and 7, however, are more practical. They offer drills and exercises to help students practise some of the theoretical concepts introduced in Modules 1, 2, 3 and 4, and apply them to different text genres. With this in mind, each module starts with an overview of the genre in question and is followed with drills and exercises to help learners practise the translation strategies outlined. Before they are tackled in detail, Module 1 will cover the common strategies of translation in order to acquaint learners with the various ways they can translate their texts.

The modules are:

\section{Foundation Methods of Translation}

This module introduces students to some of the main translation approaches and frameworks that students would find useful in translating different texts. These approaches are not comprehensive but representative of the main translation approaches used in the field.

\section{Literary Texts}

This module introduces students to literary texts and literary translation from English into Arabic, covering poetry, fiction and drama. Characteristics of each type of literary text are illustrated along with translated examples, mainly from English into Arabic.

\section{Economics Texts}

This module deals with the translation of texts on economics and finance as well as other issues dealt with in business language.

\section{Scientific Texts}

This module deals with the translation of scientific texts in areas such as medicine, physics, chemistry and psychology. Learners are shown how to deal with scientific discourse using the appropriate terminology and linguistic structures.

\section{Media Texts}

This module introduces the learner to a range of media texts. The main objective is to allow learners to familiarise themselves with the media register and to refine their translation skills. The module also introduces the main features of media texts.

\section{Administrative Texts}

Modules 6 and 7 are related. This module introduces students to administrative texts and provides learners with glossaries to help with their translation of texts in this genre. 


\section{Legal Texts}

This module introduces learners to the main features and characteristics of legal texts including civil law documents, business contracts, court documents and orders, international legal documents and resolutions, civil partnership documents, international treaties and conventions. A general introduction to the main features of legal texts, as well as the challenges of translating within this genre, is also provided. 